\title{
COVID-19 and the decolonization of Indigenous public health
}

\author{
Lisa Richardson MD MA, Allison Crawford MD PhD
}

Cite as: CMAJ 2020 September 21;192:E1098-1100. doi: 10.1503/cmaj.200852

I

ndigenous Peoples of Turtle Island, the land now known as North America, have suffered waves of infectious diseases since the arrival of Europeans more than 500 years ago, including small pox, influenza A ("Spanish flu"), tuberculosis (TB), influenza A subtype H1N1 and now severe acute respiratory syndrome coronavirus 2 (SARS-CoV-2). Although public health measures such as social distancing and the adoption of hygiene practices have necessarily been the mainstay of Canada's approach to containing coronavirus disease 2019 (COVID-19), these measures require people to have housing, water, food and income security, which are often inadequate in Indigenous communities. Such deficiencies in basic determinants of health have led to global calls for health-equity approaches to COVID-19 that urgently address the social determinants of health in Indigenous communities. ${ }^{1,2}$ However, a robust Indigenous public health response in Canada must also reconcile with the country's colonial history and uphold the self-determination of Indigenous Peoples.

The World Health Organization (WHO) defines social determinants of health as "the conditions in which people are born, grow, live, work and age." These are related to the "distribution of money, power and resources," which is driven by policy choices. The adverse health experiences of Indigenous Peoples in Canada are not only linked to social determinants, but also to systemic racism in society and the health system, which results in barriers to accessing care and care that does not meet expected quality standards. ${ }^{3}$ Such determinants resulted in high rates of infections among Indigenous Peoples in Canada during previous epidemics. They underpin the observed disproportionate rates of COVID-19 seen among people of the Navajo Nation in the United States ${ }^{4}$ and Brazilian Indigenous Peoples. ${ }^{5}$ However, they are inseparable from an important proximal determinant: colonization.

In Canada, historical colonial practices included the creation of the reserve system, the outlawing of Indigenous cultural practices, the removal of children from their families to attend residential schools, the Sixties Scoop and many other legislated policies with goals to assimilate or eradicate Indigenous Peoples. ${ }^{6}$ Medicine and public health have been an accompanying tool of colonization, particularly in Indigenous communities, as exemplified in North American and Australian scholarship..$^{7-10}$ Medical interventions and public health practices emerged to deal with the infections that

\section{KEY POINTS}

- Social equity, including adequate housing, water, food and income, is necessary to enable people and communities to employ public health measures during the current pandemic.

- Public health approaches must be implemented with awareness of how colonization has affected health care experiences and public health practices for Indigenous Peoples.

- Despite elevated rates of coronavirus disease 2019 (COVID-19) among other Indigenous communities globally, and despite our colonial history within health care and ongoing social inequities, the rate of COVID-19 for Indigenous communities in Canada is lower than that for the general population.

- Indigenous self-determination, leadership and knowledge have been successful in protecting Indigenous communities in Canada during the COVID-19 pandemic, and these principles should be at the forefront when planning public health approaches with Indigenous Peoples.

settlers brought with them. Quarantine established the "clean" perimeters of community, what was normative and what must be excluded. Many of these practices stifled local Indigenous healing practices. Indigenous strengths and medical knowledge were ignored, and Indigenous people were often depicted as primitive, childish and dirty - the antithesis of healthy. Such racist attitudes and paternalism rationalized public health approaches that diminished Indigenous rights and sovereignty. ${ }^{9}$ Across Canada, Indigenous Peoples were relocated outside of their communities for treatment and segregated into Indian hospitals. ${ }^{8,10}$

Yet colonialism is not only a historical phenomenon; its forces and structures continue to affect the well-being of Indigenous Peoples worldwide, underpinning many adverse social determinants of health experienced by Indigenous Peoples but also disrupting access to protective determinants such as language, traditional governance, land-based activities and healing practices. The Truth and Reconciliation Commission's Calls to Action enjoin Canadians to acknowledge the effects of colonialism and ongoing health inequities in our health care system. ${ }^{6}$ Historical medical and public health practices contribute to contemporary social suffering and continue to affect the way that First Nations, Inuit and Métis communities 
view public health and public health recommendations. A qualitative study of people with TB in Nunavut, for example, found that previous experiences with public health approaches to treating tuberculosis in the 1950s deterred some Inuit from seeking treatment for tuberculosis quite recently, ${ }^{10}$ with 1 participant describing feelings of lack of respect and autonomy that affected their current adherence to public health recommendations: "Health care people do not treat patients with respect. It is as if they don't care to explain anything, like the patients won't understand anyway and the health care people know better. People are generally not told about things. They are not informed so that they can have a choice. The doctors or nurses choose for them." 10

Although public health historically became a tool of colonization, several key concepts in contemporary public health practice resonate with approaches to health and well-being that exist in diverse First Nations, Inuit and Métis communities. Anishinaabe health concepts, for example, include a focus on community and on the relationship between a community and the land, a recognition of

\section{INDIGENOUS WELLBEING in the Times of C满VID-19: Four Directions Virtual Support Hub}
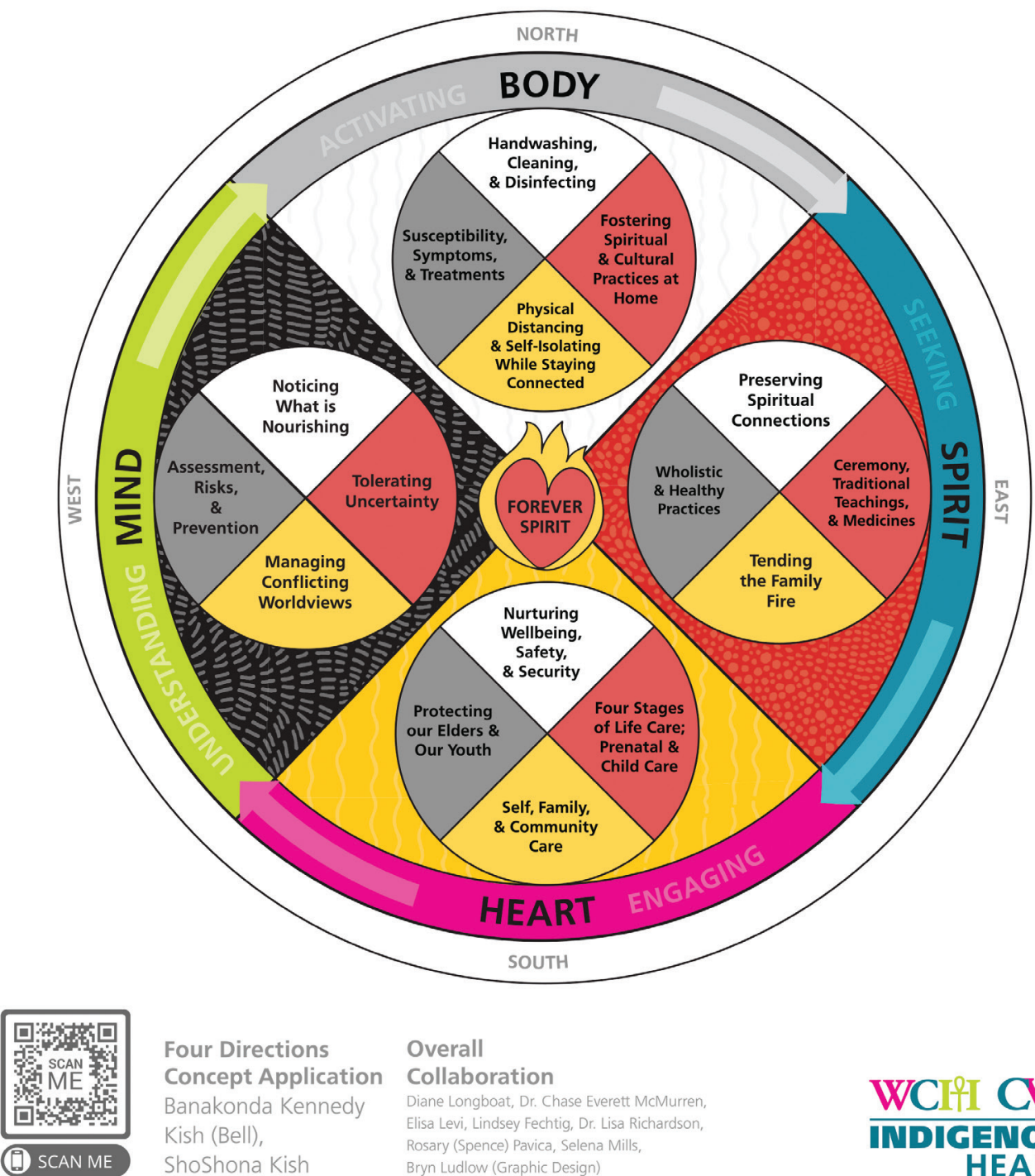

Four Directions

Overall

Concept Application

Banakonda Kennedy

Kish (Bell),

ShoShona Kish

\section{Collaboration}

Diane Longboat, Dr. Chase Everett McMurren

Elisa Levi, Lindsey Fechtig, Dr. Lisa Richardson

Rosary (Spence) Pavica, Selena Mills,

Bryn Ludlow (Graphic Design)

\section{WCITI CWP INDIGENOUS HEALTH}

Figure 1: Indigenous well-being during COVID-19 (see www.womenscollegehospital.ca/research,-education-and-innovation/indigenous-wellbeing-in -the-times-of-covid-19). Permission to reprint this figure is granted by The Centre for Wise Practices in Indigenous Health, Women's College Hospital, Toronto. Note: COVID-19 = coronavirus disease 2019. 
dimensions of health beyond the physical and the mental, and an understanding of health as a state of well-being rather than the absence of disease. This corresponds somewhat with contemporary Western understandings of public and global health, offering promise of potential alignment between Indigenous public health practice and key principles of Western public health such as contact tracing or preventive testing strategies.

A successful adaptation or integration of Western approaches for Indigenous public health must be anchored in the principles of self-determination for Indigenous Peoples as articulated in the 2007 United Nations Declaration on the Rights of Indigenous People to determine their own paths to healing and to health for their communities.

In Canada, almost all Indigenous communities have pre-existing emergency preparedness plans, and they have been updated and implemented to deal with the current pandemic. Furthermore, innovative educational materials and public health campaigns have been created by many different First Nations, Inuit and Métis communities. These materials build on prevention, isolation and containment measures put forward by public health institutions but are grounded in the local context of each community, including its language, culture, and physical and social environments. Examples include the Qikiqtani Inuit Association's educational materials, which illustrate hygiene practices, social distancing and isolation procedures in Inuktitut, using common examples from the daily lives of their local community members, as well as frameworks for wellness in the COVID era that draw on the knowledge of Elders and knowledge keepers from specific nations. The adaptation by the Centre for Wise Practices at Women's College Hospital in Toronto of the Four Directions Medicine Wheel for a holistic approach to preventing and building physical, emotional, mental and spiritual resilience during COVID-19 (Figure 1), is an example of the latter.

During COVID-19 many Indigenous communities have shown self-determination by articulating and enforcing rules on who can enter their communities, often implementing far stricter measures than those enacted by local municipalities, such as closures and checkpoints. Furthermore, globally ${ }^{11}$ and in Canada, for example, in the province of Manitoba, Indigenous leaders have advocated for the collection of Indigenous-specific COVID data with clear data sovereignty agreements related to access, control, ownership and possession of the data.

In contrast to predictions early in the course of COVID-19, First Nations, Inuit and Métis communities in Canada have had lower rates of COVID-19 overall and a lower case fatality rate than nonIndigenous Peoples despite structural inequities and social determinants that are generally related to poorer health outcomes. ${ }^{12}$ First Nations on-reserve have had a COVID-19 case rate 4 times lower than that of the general Canadian population, with 3 times fewer fatalities and a $30 \%$ higher recovery rate. ${ }^{12}$ This is vastly different from outcomes during the $\mathrm{H} 1 \mathrm{~N} 1$ pandemic where rates of the infection were 1000 per 100000 population among Inuit, and 24 per 100000 population among non-Indigenous people in Canada. ${ }^{13}$ Current strategies for Indigenous public health practice, grounded in self-determination, are an important reason for the relative resilience of Indigenous communities in Canada during COVID-19.
Anticipating further waves of COVID-19, it is important that the design, implementation and leadership of public health by First Nations, Inuit and Métis communities continue in Canada. At its foundation, Indigenous public health must be self-determined: adapted for the needs of specific nations and grounded in local Indigenous language, culture and ways of knowing; developed, implemented and led by Indigenous Peoples; and informed by ongoing monitoring of data as governed by appropriate data sovereignty agreements. Moreover, all levels of government in Canada must address the social determinants of health both in the short term - to facilitate prevention, control and containment of COVID-19 - and in the long term through investments in infrastructure, food security and chronic disease prevention and management. This will require the decolonizing of health care at individual, organizational and policy levels. Governments, policymakers and public health providers must embrace the knowledge, expertise and strong leadership of Indigenous communities to face COVID-19.

\section{References}

1. Ferrante L, Fearnside PM. Protect Indigenous peoples from COVID-19. Science 2020;368:251.

2. Curtice K, Choo E. Indigenous populations: left behind in the COVID-19 response. Lancet 2020;395:1753.

3. Allan B, Smylie J. First Peoples, second class treatment: the role of racism in the health and well-being of Indigenous Peoples in Canada. Toronto: the Wellesley Institute; 2015.

4. Kovich H. Rural matters: coronavirus and the Navajo Nation. N Engl J Med 2020;383:105-7.

5. Cupertino GA, do Carmo Cupertino M, Gomes AP, et al. COVID-19 and Brazilian Indigenous populations. Am J Trop Med Hyg 2020;103:609-12.

6. Truth and Reconciliation Commission of Canada: calls to action. Winnipeg: Truth and Reconciliation Commission of Canada, 2012; 2015. Available: http:// trc.ca/assets/pdf/Calls_to_Action_English2.pdf (accessed 2020 Apr. 15).

7. Anderson WP. The cultivation of whiteness: science, health and racial destiny in Australia. Melbourne (AU): Melbourne University Press: 2005.

8. Lux MK. Separate beds: a history of Indian hospitals in Canada, 1920s-1980s. Toronto: University of Toronto Press; 2016.

9. Crawford A. Project CREATeS: youth engagement in suicide prevention. Lancet 2019;394:1222-3.

10. Møller H. Tales about tuberculosis and colonization: the socio-cultural experience of tuberculosis in Nunavut. Alaska Med 2007;49(Suppl 2):179-83.

11. Zavaleta C. COVID-19: review Indigenous Peoples' data. Nature 2020;580:185.

12. Update on COVID-19 in Indigenous communities [news release]. Indigenous Services Canada; 2020 July 7. Available: www.canada.ca/en/indigenous-services -canada/news/2020/07/update-on-covid-19-in-indigenous-communities.html (accessed 2020 July 30 ).

13. The 2009 HINI influenza pandemic among First Nations, Inuit and Métis peoples in Canada: epidemiology and gaps in knowledge. National Collaborating Centre for Aboriginal Health. Prince George (BC): National Collaborating Centre for Aboriginal Health; 2016. Available: www.ccnsa-nccah.ca/docs/other/FS -InfluenzaEpidemiology-EN.pdf (accessed 2020 July 30).

\section{Competing interests: None declared.}

This article has been peer reviewed.

Affiliations: Departments of Medicine (Richardson) and Psychiatry (Crawford), University of Toronto; Centre for Addiction and Mental Health (Crawford); University Health Network (Richardson), Toronto, Ont.

Contributors: Both authors contributed to the conception and design of the work, drafted the manuscript, revised it critically for important intellectual content, gave final approval of the version to be published and agreed to be accountable for all aspects of the work.

Correspondence to: Allison Crawford, allison.crawford@utoronto.ca 